
\title{
25 Research Suare \\ Biomarkers predictive of long-term fertility found in vaginal lipidome of gilts at weaning
}

\section{Kayla M Mills}

Purdue University College of Agriculture

Christina R Ferreria

Purdue University

Jebadiah Stevens

Ag Production Enterprise

Kara R Stewart ( $\boldsymbol{\nabla}$ krstewart@purdue.edu )

Purdue University College of Agriculture https://orcid.org/0000-0002-6223-8159

Theresa M Casey

Purdue University College of Agriculture

\section{Research}

Keywords: lipidome, fertility, gilt development, pig

Posted Date: February 19th, 2021

DOI: https://doi.org/10.21203/rs.3.rs-210669/v1

License: (우 (i) This work is licensed under a Creative Commons Attribution 4.0 International License. Read Full License 


\section{Abstract \\ Background}

A marker indicative of fertility potential of replacement gilts early in development would decrease culling rates in the sow herd, improve sow herd reproductive efficiency, and reduce production costs. The objective of this study was to determine if vaginal lipid profiles at $21 \mathrm{~d}$ postnatal (PN) could predict sow reproductive performance.

\section{Methods}

Vaginal swabs of the anterior vagina were taken at $21 \pm 4 \mathrm{~d}$ PN from gilts born on a commercial sow production facility for lipidomic analysis. Animals were followed prospectively for two years and assigned to reproductive performance categories based on observation of estrus or piglets weaned per sow per year (PSY) across two farrowings.

\section{Results}

Lipids were extracted from cellular material collected with swabs taken from high fertility (HF; $n=28 ; \geq 26 P S Y)$ and infertile (IF; $n=34$; no estrus, no pregnancy) animals and multiple reaction monitoring (MRM) profiling was used for lipidome analysis. Relative abundance of arachidonic acid (ARA, C20:4) and docosahexaenoic acid (DHA, C22:6) were lower $(P<0.05)$ in IF gilts than HF gilts, whereas abundance of the free fatty acids cerotic (C26:0), ximenic (C26:1), and nonadecanoic (C19:0) acids were greater $(P<0.05)$ in IF gilts. Additionally, eicosapentaenoic acid (C20:5) a precursor of prostaglandins was also higher $(P<0.05)$ in IF gilts.

\section{Conclusions}

The perspective of having a panel of lipids captured with vaginal swabs at weaning that can predict reproductive efficiency of gilts shows promise and warrants future research in this area.

\subsection{Background}

Sow's reproductive efficiency has a major effect on economic profitability in the swine industry. A common method to measure reproductive efficiency is number of piglets weaned per sow per year (PSY) (1-3). In 2018, the average PSY for US sow herds was 25.3 (4). Another way to measure farm reproductive efficiency is sow replacement rate. Despite high yielding sows, the annual removal rates of animals from herds averaged $45 \%$ across the US in 2018, with culling primarily due to poor reproductive performance. The high culling rate of sows from herds is due, in part, to the lack of the ability of the producer to identify reproductively sound gilts early in life.

To maximize economic returns and efficiency for the farm there is a need to identify animals with the greatest reproductive potential as early in their lifetime as possible. An efficient gilt management system has three selection timepoints: at weaning, gilts selected to receive boar exposure, and from this group, gilts identified as exhibiting estrus (5). Minimization of differences in size and growth rates of animals at 21 days of age due to 
perinatal management practices like cross fostering to equalize piglet access to sow nipples, and thus milk during lactation, make the initial step of replacement gilt selection at weaning challenging. Currently, the most predictive parameter of sow reproductive efficiency occurs at the second stage and is average age at first estrus. However the second stage is more than 200 days into the production timeline and the producer has already invested in an animal that may fail to show estrus (5).

Studies aimed at identifying markers of fertility found that vulva width at 95-115 days of age associated with a gilt's ability to achieve puberty by 200 days of age (6), and vulva width at 105 days was associated with sow productivity through two parities (7). These findings indicate that development of the lower reproductive tract reflects reproductive efficiency and fertility, and thus support exploration of early stage phenotypes and biomarkers for identification of replacement gilts for sow herds. In our previous study, we evaluated whether phenotypic traits, such as birth weight, weaning weight, vulva length, vulva width, birth and nursing litter size were related to reproductive performance later in life (8). We found that vulva width at 21 days of age was predictive of whether or not a gilt would become pregnant later in life, however, this relationship was weak and vulva width only explained $1.5 \%$ of the variation in reproductive success (8).

A link between perinatal nutritional environment with uterine development and long-term fertility has also been established for swine (9-14) Therefore, we conducted additional studies to explore if there were any associations between the vaginal lipidome and perinatal nutrition $(15,16)$. Vaginal swabs taken on $d 14 \mathrm{PN}$ revealed that certain glycerolipids containing arachidonic acid (C20:4) were potential biomarkers for colostrum intake when gilts were either fed colostrum or milk replacer (16). In a follow-up study, we found the vaginal lipidome on d 2 PN of gilts fed milk replacer reflected the lipids present in milk replacer, thus indicating that the nutrition in the perinatal environment influences the composition of the reproductive tract and could reflect the gilt's reproductive success later in life (15). Therefore, the objective of this study was to determine whether there were lipid biomarkers in cellular material captured with vaginal swabs taken at weaning predictive of lifetime sow productivity. Specifically, multiple reaction monitoring (MRM) profiling was used to profile lipids collected with vaginal swabs at 21 days postnatal (PN) and the relationship of this profile with their fertility at maturity was explored.

\subsection{Materials And Methods}

\subsubsection{Animals}

All procedures involving animals were reviewed and approved by the Institutional Animal Care and Use Committee at Purdue University prior to beginning the study. Animals used were a subset of a larger previously described study (8). Briefly, gilts $(\mathrm{n}=2146)$ born on a commercial farm in central Indiana were enrolled in a longitudinal observational study (Figure 1). Initial individual weights were recorded between d 0 and 2 postnatal and are referred to hereafter as birth weights. Piglets were weighed and processed on postnatal d 2-3 (ear tag, $200 \mathrm{mg}$ iron, tail docking, antibiotic administration) as per routine management on the farm. Cross-fostering between litters of similar aged piglets occurred after piglets were given an ear tag (litter size standardized to $14 \pm$ 1 piglets). Data on farrowing date, birth litter size, size of gilt's litter at her weaning, birth weight, and weaning weight were entered into MetaFarms (MetaFarms, Inc., Burnsville, MN) database for each gilt.

Gilts ( $n=1084$ ) were weaned at $21 \pm 4 \mathrm{~d}$, selected as potential replacement sows, and moved to the farm's onsite nursery (Figure 1). Gilts that were not selected as replacement females were transferred to an offsite wean to 
finish facility for market production. Immediately following selection into the onsite nursery, swabs of the anterior vagina were taken for lipidomic analysis. Gilt's vulva was sprayed with ethanol and wiped clean with gauze before the swab was taken. A human pap smear brush (Rovers ${ }^{\circledR}$ EndoCervex-Brush ${ }^{\circledR}$, Oss, Netherlands) was placed into the vagina as far as possible and then rotated clockwise to get a representative scraping of the anterior vagina. Swabs were taken in duplicate and then placed in $15 \mathrm{~mL}$ polypropylene conical tubes (Corning ${ }^{\text {TM }}$ Falcon $^{\mathrm{TM}}$, Corning, NY) and immediately placed on ice, and transferred to the Purdue laboratory and stored at $-80^{\circ} \mathrm{C}$ until analysis.

Gilts ( $n=400)$ approaching 25 weeks of age were selected to move from the on-site nursery into the gilt development unit (GDU) to receive daily, full-contact boar exposure to induce puberty. Following observance of a second estrus, gilts were moved to a gestation crate to be bred using artificial insemination (Al) on their third estrus. Gilts failing to show estrus by 28 weeks of age were administered a full dose of P.G. 600 (Intervet America, Inc., Millsboro, DE) and bred as soon as they came into estrus. Gilts that never showed estrus were removed from the selection pool. Breeding date, age of first estrus, and induction of estrus with P.G. 600 were entered into the Metafarms database.

\subsubsection{Categorization of Fertility Groups}

Performance data from breeding herd animals $(n=400)$ were extracted from Metafarms reports which included date of birth, dates of estrus detection, herd removal (cull) date, reason for removal, farrowing date and litter information (total born, number born alive, preweaning mortality). Gilts that were culled from the breeding herd for non-reproductive reasons were removed from data set. Gilts that remained $(n=353)$ were divided into fertile and infertile groups (Figure 1). Fertile animals were classified into one of three subclasses: sows with at least 26 PSY were defined as High Fertility (HF; n=82), 20-25 PSY were classified as Middle Fertility (MF; n=43), and less than 20 PSY were classified as Low Fertility (LF; n=54). PSY was chosen as it encompasses multiple facets of fertility and prolificacy, such as ovulation rate, wean to estrus interval, farrowing rate, and embryo survival rate (17-19). Infertile animals were divided into two subclasses based on whether they exhibited estrus following routine boar exposure or P.G. 600 administration. Infertile-Estrus (IFe; $n=36$ ) were gilts that showed signs of estrus but did not become pregnant and Infertile-No Estrus (IFno; $n=138$ ) were gilts that never exhibited signs of estrus. A subset of the most fertile (HF; $n=28)$ and infertile animals that did not exhibit estrus (IF; $n=34)$ were randomly selected for lipidome analysis.

\subsubsection{Statistical analysis of phenotypic traits}

Analysis of phenotypic traits such as birth litter size, nursing litter size, average daily gain from birth to weaning, birth weight, weaning weight, and vulva width by fertility category was performed using the GLM procedure of SAS 9.4 (SAS Inst. Inc., Cary, NC).

\subsubsection{Lipidomic Extraction and Multiple Reaction Monitoring (MRM) Profiling}

Lipids were extracted from swabs using the Bligh and Dyer method (20). Samples were thawed at room temperature before the start of the extraction. Swabs were rinsed with $500 \mu$ l deionized water and vortexed to remove cellular material in the same $15 \mathrm{ml}$ polypropylene conical tubes. Two hundred $\mu \mathrm{l}$ of sample was transferred to a new $1.7 \mathrm{ml}$ tube (Axygen ${ }^{\circledR}$, Corning, New York). Four hundred fifty $\mu$ l of methanol prepared with $1 \mathrm{mM}$ butylated hydroxytoluene and $250 \mu \mathrm{l}$ of chloroform was added to the solution and vortexed. Samples were 
then incubated for $15 \mathrm{~min}$ at $4^{\circ} \mathrm{C}$. An additional $250 \mu \mathrm{l}$ of both deionized water and chloroform were added to the tube and centrifuged $10 \mathrm{~min}$ at $3000 \mathrm{rcf}$ at $4^{\circ} \mathrm{C}$. The solution was separated into three phases consisting of the polar, protein, and organic (lipid) phase. The lipid phase was removed, placed into $1.7 \mathrm{ml}$ microcentrifuge tubes, and dried in a vacuum concentrator for $8 \mathrm{~h}$. Dried pellets were resuspended $200 \mu \mathrm{l}$ of acetonitrile, methanol, and ammonium acetate 3:6.65:0.35 (v/v/v). Further 10X dilution of sample in solvent was used for direct injection.

Multiple reaction monitoring (MRM) profiling was done using a two-part process beginning with a discovery phase followed by a screening phase. The discovery phase was used to determine which lipids were detectable in the samples. For this, ten $\mu \mathrm{l}$ from each sample was pooled by phenotype into a $1.7 \mathrm{ml}$ tube and then dried by nitrogen flow for $8 \mathrm{~h}$. Dried lipid extracts were diluted in $200 \mu \mathrm{l}$ of acetonitrile, methanol, and ammonium acetate 3:6.65:0.35 ( $\mathrm{v} / \mathrm{v} / \mathrm{v})$. Eight $\mu \mathrm{l}$ from each pooled sample were injected into the microautosampler $(\mathrm{G} 1377 \mathrm{~A})$ in a QQQ6410 triple quadrupole mass spectrometer (Agilent Technologies, San Jose, CA) equipped with an ESI ion source. A solvent solution containing acetonitrile with $1 \%$ formic acid at $10 \mu \mathrm{l} / \mathrm{min}$ was pumped between injections (CapPump G1376A, Agilent Technologies, San Jose, CA). Pure methanol was injected between samples to remove any remaining lipids from the previous injection.

During the discovery phase, pooled samples were screened for the chemical classes of acylcarnitine (AC), cholesteryl ester (CE), ceramide, free fatty acids (FFA), phosphatidylcholines (PC), phosphatidylethanolamine (PE), phosphatidylinositol (PI), phosphatidylglycerol (PG), phosphatidylserine (PS), and triacylglycerol (TAG) using MRM profiling methods previously reported (21-24). Additionally, lipids from previous research that had been found by MRM profiling to discriminate between gilts fed different postnatal diets were also screened (25). Our studies described in Harlow et al., 2019, identified 146 lipids that discriminated between gilts that suckled colostrum versus bottle fed milk replacer the first $48 \mathrm{~h}$ postnatal, and are referred to as Method 1 (15). Lipids in Method 1 were primarily TAG, PE, PC, and PG. A second set of 197 lipids distinguished between gilts fed a lard based fat supplementation and unsupplemented animals, and are referred to as Method 2 (15). Lipids in Method 2 were primarily glycerolipids (15). Processing the initial chemical class data was completed using MSConvert20 which converted each set of profiling method data into mzML format. Signal intensity for ions present in the samples was obtained using an inhouse script. lons with values $>30 \%$ in at least one of the samples compared to a blank within each profiling method were selected for the screening phase.

1. Due to the large number of MRMs to be screened, the samples were interrogated using four lists of MRMs, which we refer as methods:

2. Phosphatidylcholines (PC) (Supplemental Table 1)

3. Free fatty acids (FFA) (Supplemental Table 2)

4. Lipid classes defined by Harlow et al., as Method 1 (Supplemental Table 3)

5. Lipid classes defined by Harlow et al., as Method 2 (Supplemental Table 4)

\subsubsection{MRM profiling data analysis in MetaboAnalyst}

For analysis, relative intensity of a given MRM in each sample was calculated by class of lipid or method employed in each screen, following removal of MRM ion pairs with intensities less than 1.3-fold of the blank sample. Relative intensity of MRM ion pairs was calculated by dividing intensity by the sum of intensities of all lipids within a sample by screening method. Relative intensity of MRM ion pairs were uploaded into MetaboAnalyst 3.0 (26) and data were normalized using autoscaling. Student t-test analysis was used to identify 
MRMs that distinguished between fertility phenotypes, using an alpha of 0.05 of nominal $P$-value to identify differentially distributed lipids. Biomarker analysis was completed using classical univariate receiver operating characteristic (ROC) curve analysis with area-under-the-curve (AUC) value used to determine a lipid's potential as a biomarker. The following AUC scale was used to evaluate lipids as potential biomarkers: excellent = 0.9-1.0; good=0.8-0.9; fair=0.7-0.8; poor=0.6-0.7; fail=0.5-0.6 (27).

\subsection{Results}

There were no differences between highly fertile (HF) and infertile (IF) gilts in their birth and weaning weights, nor their birth or suckling litter size (Table 1). Moreover, there was no difference in vulva size at weaning, which is consistent with our previous findings of the entire population (8).

Table 1 Birth and weaning weight, litter size and vulva width at weaning of high fertility (HF) and infertile (IF) gilts.

\begin{tabular}{|lllll|}
\hline & HF & IF & SE & $p$-value \\
& $(\mathrm{n}=28)$ & $(\mathrm{n}=34)$ & & \\
\hline birth litter size, piglets & 13.04 & 12.82 & 0.49 & 0.74 \\
\hline nursing litter size, piglets & 13.54 & 13.41 & 0.14 & 0.53 \\
\hline average daily gain ${ }^{1}, \mathrm{~kg} / \mathrm{d}$ & 0.16 & 0.16 & 0.01 & 0.96 \\
\hline birth weight, $\mathrm{kg}$ & 1.77 & 1.67 & 0.06 & 0.20 \\
\hline weaning weight, kg & 5.29 & 5.12 & 0.29 & 0.65 \\
\hline vulva width, $\mathrm{mm}$ & 9.00 & 8.81 & 0.29 & 0.65 \\
\hline
\end{tabular}

${ }^{1}$ Average daily gain from birth to weaning

The MRM profiling discovery phase identified a total of 269 MRMs related to distinct molecules in pooled samples from HF and IF vaginal swabs. Phosphatidylcholines (PC) accounted for $30 \%$ of the MRMs identified followed by free fatty acids (FFA) at 13\% (Figure 1; Supplemental Table 1). MRMs identified in the discovery phase along with lipids previously identified as distinguishing gilts based on perinatal nutrition (Method 1 and 2; Harlow et a., 2019) were profiled in individual samples during the screening phase. Tentative attributions were assigned to differentially abundant lipids within each lipid class (Table 2). There were no distinguishing lipids identified using the PC method. Out of 36 FFA lipids, six were different between HF and IF gilts (Table 2). Arachidonic acid (ARA) and docosahexaenoic acid (DHA) were higher $(P<0.05)$ in HF than IF gilts, whereas cerotic acid $C(26: 0)$, ximenic acid $C(26: 1)$, nonadecanoic acid (C:19:0), and pentadecanoic acid (C15:0) were lower $(P<0.05)$ in HF than IF gilts. Of the 181 lipids screened by Method 1, 12 were differentially abundant $(P<0.05)$ between HF and IF gilts. HF had a greater amount of MRMs attributed as PC(32:1) and SM(d18:0/18:0). Infertile animals had a greater amount of MRMs related to PC(40:6), PCo(38:0), cholesterol ester 18:0, docosadienoylcarnitine, PC(40:7), PS(32:2), PC(40:6), Lyso-PG(20:5), PSo(18:0), and PC(40:8). Only one of the 102 MRMs in Method 2 was different $(P<0.05)$ between HF and IF animals and was the unidentified MRM of 554.79 -> 282.192, which was higher $(P<0.05)$ in HF compared to IF animals. 
ROC curve analysis was performed to determine the potential of lipids differentially abundant between HF and IF as biomarkers of fertility (Table 2; Figure 2). Cerotic acid (C26:0), ximenic (C26:1), and nonadecanoic acid (C19:0) had AUC between 0.7-0.8, indicating fair potential as biomarkers. All other distinguishing lipids had an AUC of $0.6-0.7$, indicating poor potential as biomarkers of fertility status (Table 2).

Table 2. Relative difference between HF and IF in abundance of lipids extracted from vaginal swabs taken at 21 days of age.

\begin{tabular}{|c|c|c|c|c|c|c|}
\hline Method & $\begin{array}{l}\text { Tentative ID } \\
\text { Attribution/MRM } \\
\text { ID }\end{array}$ & Lipid Class & Common Name & $\begin{array}{l}\text { HF-IF } \\
\text { FC }^{1} \\
(\log 2)\end{array}$ & $p$-value & AUC $^{2}$ \\
\hline \multirow[t]{6}{*}{ FFA } & $C(26: 0)$ & free fatty acid & Cerotic Acid & -0.30 & $<0.01$ & 0.74 \\
\hline & $C(26: 1)$ & free fatty acid & Ximenic Acid & -0.43 & 0.01 & 0.71 \\
\hline & $C(19: 0)$ & free fatty acid & Nonadecanoic acid & -0.16 & 0.01 & 0.73 \\
\hline & $C(20: 4)$ & free fatty acid & Arachidonic Acid & 0.28 & 0.03 & 0.66 \\
\hline & $C(15: 1)$ & free fatty acid & Pentadecenoic acid & -0.25 & 0.04 & 0.64 \\
\hline & $C(22: 6)$ & free fatty acid & $\begin{array}{l}\text { Docosahexaenoic } \\
\text { Acid }\end{array}$ & 0.41 & 0.05 & 0.60 \\
\hline \multirow[t]{11}{*}{ M1 } & $\mathrm{PC}(40: 6)$ & Glycerophosphocoline & $\begin{array}{l}\text { Phosphatidylcholine } \\
40: 6\end{array}$ & -0.17 & 0.01 & 0.68 \\
\hline & PCo(38:0) & Glycerophosphocoline & $\begin{array}{l}\text { Phosphatidylcholine } \\
\text { 0-38:0 }\end{array}$ & -0.23 & 0.02 & 0.64 \\
\hline & CE(18:0)/ST(16:0) & $\begin{array}{l}\text { Cholesterol } \\
\text { ester/Sitosteryl ester }\end{array}$ & $\mathrm{CE}(18: 0) / \mathrm{ST}(16: 0)$ & -0.30 & 0.02 & 0.65 \\
\hline & $\mathrm{PC}(32: 1)$ & Glycerophosphocoline & $\begin{array}{l}\text { Phosphatidylcholine } \\
(32: 1)\end{array}$ & 0.16 & 0.03 & 0.65 \\
\hline & $\mathrm{PC}(40: 7)$ & Glycerophosphocoline & $\begin{array}{l}\text { Phosphatidylcholine } \\
(40: 7)\end{array}$ & -0.17 & 0.03 & 0.64 \\
\hline & PS(32:2) & Glycerophosphoserine & PS(32:2) & -0.31 & 0.03 & 0.63 \\
\hline & $\mathrm{PCo}(40: 6)$ & Glycerophosphocoline & $\begin{array}{l}\text { Phosphatidylcholine } \\
0-40: 6\end{array}$ & -0.16 & 0.04 & 0.65 \\
\hline & LPG(20:5) & Glycerophosphoglycerol & Lyso PG 20:5 & -0.30 & 0.04 & 0.63 \\
\hline & PSo(18:0) & Glycerophosphoserine & PS 0-18:0 & -0.31 & 0.05 & 0.62 \\
\hline & PC(40:8) & Glycerophosphocoline & $\begin{array}{l}\text { Phosphatidylcholine } \\
40: 8\end{array}$ & -0.14 & 0.05 & 0.62 \\
\hline & $S M(d 18: 0 / 18: 0)$ & Sphingomyelin & $\begin{array}{l}\text { Sphingomyelin } \\
\text { d18:0/18:0 }\end{array}$ & 0.14 & 0.05 & 0.62 \\
\hline M2 & 554.79 -> 282.192 & Not Identified & $n / a$ & 0.19 & 0.02 & 0.66 \\
\hline
\end{tabular}


${ }^{1}$ Fold change

${ }^{2} \mathrm{AUC}=$ area under the curve from receiver operator characteristic (ROC) curve analysis to evaluate potential as a biomarker to distinguish animals based on fertility group.

Correlation analysis of distinguishing lipids revealed that ARA had an inverse relationship $(P<0.05)$ to cerotic acid $(r=-0.57)$, nonadecanoic acid $(r=-0.35)$, and ximenic acid $(r=0.50)$. Approximately one-third of IF animals exhibited a lipidome signature of low ARA coupled with high levels of cerotic, nonadecanoic, and ximenic acid. Ximenic acid, cerotic acid, and nonadecanoic acid had a positive relationship to each other, with ximenic and cerotic acid having a very strong relationship to each other $\left(r^{2}=0.94\right)$. It was also observed that IF animals with high levels of cerotic acid also had high levels of Lyso PG $(20: 5)\left(r^{2}=0.41\right)$. DHA had a relatively weak relationship when compared to all other lipids in the analysis $\left(-0.15 \leq r^{2} \geq 0.2\right)$. The phenotype driving the correlation between cerotic acid, ximenic acid, and ARA was the IF group (Figure 3).

\subsection{Discussion}

Our overall aim was to determine whether lipids captured from vaginal swabs taken at weaning could be used to predict fertility outcomes in swine. Relative abundance of multiple MRMs related to lipids were differentially abundant between gilts that were later found to be infertile and high fertility sows. Vaginal lipid profiles of approximately one-third of the infertile animals at weaning were found to be high in cerotic, ximenic, nonadecanoic, and eicosapentaenoic acids and low in arachidonic and docosahexaenoic acids. Arachidonic (ARA) and docosahexaenoic (DHA) acid levels in vaginal swabs were higher in gilts that were high fertility at maturity. ARA and DHA are classified as polyunsaturated fatty acids (PUFAs) and are known to be positively related to female reproductive success (28-32). Linoleic acid (LA) is the precursor to ARA and alpha linolenic acid (ALA) is the precursor of DHA. Neither LA nor ALA can be synthesized by the body and must be consumed in the diet (33). DHA and ARA can also be derived from the diet and transferred through colostrum and milk to the tissues of piglets (34-36). ARA levels are high in milk (37). In gilts fed colostrum versus milk replacer, gilts that received colostrum had a greater amount of ARA in the vaginal lipidome on PN d 14 signifying ARA as a marker of colostrum intake (16). The level of colostrum intake in the first $24 \mathrm{~h}$ postnatal was associated with long-term fertility in swine (14). Therefore, these data together establish support for a connection between level of colostrum intake, level of ARA in vaginal lipidome and long-term fertility in swine. Level of perinatal exposure to DHA has been linked to tissue levels. DHA levels within plasma, liver phospholipids, and brain plasma membrane of two week old pigs was found related to levels in milk fed to them as neonates (34). Moreover, rats and humans fed a diet high in DHA had increased reproductive success with greater oocyte quality at an advanced maternal age (28). Holstein cows consuming DHA postpartum had an increased number of large follicles (29), increased pregnancy rate at first breeding, and a greater resumption of cyclicity following calving (30). DHA treatment of bovine granulosa cells in culture increased proliferation and progesterone secretion (31). Feeding DHA to gestating gilts and sows increased litter growth and larger corpus lutea size on the ovaries when they cycled after litters were weaned (32). Similarly, sows supplemented with DHA during lactation appeared to have improved quality of ovarian follicles and increased survivability of embryos (36). DHA has inhibitory effects on prostaglandin- $\mathrm{H}$-synthase which may be beneficial for the preparation of endometrium to maintain pregnancy (38). Thus, the relationship between DHA and fertility is well documented, and now studies need to be designed to determine if increasing exposure of gilts to DHA during the perinatal period affects their long-term fertility. 
In this study, ROC curve analysis revealed that nonadecanoic acid had the highest AUC value of all distinguishing lipids. Nonadecanoic acid is a 19-carbon saturated fatty acid that has ties to reduced fertility. Men with asthenozoospermia had higher levels of nonadecanoic acid within their seminal plasma than their healthy counterparts (39). This group found high levels of C19:0 to be a potential biomarker of asthenozoospermia related infertility in men (39). However, little is known about the relationship between elevated nonadecanoic acid and impaired female fertility. Pentadecenoic acid (C15:1) was also found to be higher in IF gilts. Odd-chain fatty acids must be broken down via alpha-oxidation within the peroxisome (40). Therefore, a buildup of odd chain fatty acids in IF gilts may be indicative of a peroxisomal issue.

ROC curve analysis indicated that cerotic and ximenic acid were fair biomarkers of infertility and were higher in vaginal swabs of IF gilts at weaning. Cerotic acid, is a saturated very long-chain fatty acid (VLCFA) consisting of 26 carbons $(41,42)$. VLCFAs with C22 and C24 are found ubiquitously throughout the body, whereas VLCFAs with 26 carbons or more are sub-classified as ultralong-chain FAs (ULCFAs) and are found in the skin, retina, testis, and brain (43). The outermost layer of the epidermis is comprised of saturated and monounsaturated UCLFAs which are essential in creating a barrier against water loss and invasion of pathogens (44). Similar to the epidermis, the lumen of anterior region of the vagina is composed of stratified epithelium. Therefore, the presence of ULCFAs captured on vaginal swabs is to be expected. However a higher proportion of them, as evident in the IF group, may be indicative of alterations in lipid metabolism that affects health and fertility potential (45). Fatty acids longer than 22 carbons are exclusively broken down in the peroxisome (46). Impairment of VLCFA degradation pathways is associated with several diseases related to impaired peroxisomal function and biogenesis (43). Cerotic acid build-up is found in individuals with adrenoleukodystrophy (ALD). ALD is an X-linked recessive condition characterized by buildup of VLCFA in tissues due to a mutation in the $A B C D 1$ gene $(47,48)$. ABCD1 functions to transport VLCFA into peroxisomes. In addition to the development of cognitive impairment, males with ALD are infertile. Accumulation of saturated fatty acids within Sertoli cells is hypothesized to be a cause of infertility in men with $\operatorname{ALD}(47,49)$.

The eicosanoid Lyso-PG (20:5) was higher in IF animals and positively related to cerotic acid levels in vaginal swabs. The fatty acid associated with Lyso-PG (20:5) is a PUFA that is converted to prostaglandins (50). Infertile gilts also had a higher abundance of certain phosphatidylcholines, namely PC(40:6), PC(40:7), PCo(40:6), and PC(40:8), which are highly unsaturated and compatible with IF animals having a buildup of eicosanoids in vaginal tissue at weaning. In a fertility trial, cows who were less receptive to pregnancy had a greater abundance of PC(40:6) and PC(40:7) in their uterine lipidome (51). Perigonadal tissue from reproductively old mice also had a higher abundance of $\mathrm{PC}(40: 6)$ and $\mathrm{PC}(40: 7)$ (22). Another disease associated with the presence of elevated VLCFAs is Zellweger syndrome. Individuals with this condition lack intact peroxisomes and cannot properly metabolize prostaglandins (52).

Lipid accumulation, or lipotoxicity, is associated with mitochondrial and peroxisomal dysfunction, endoplasmic reticulum stress, and negatively impacts fertility (53-56).

The build-up of ULCFA and eicosanoids in tissue of IF animals suggest that peroxisomal dysfunction maybe at the root of the problem. Peroxisomes are required for the first steps of beta-oxidation of ULCFA and degradation of eicosanoids $(43,57,58)$. Individuals with Zellweger syndrome have mutations in genes that encode for peroxin (PEX) proteins. Peroxins are required for peroxisome biogenesis (59), and peroxisome proliferator activator receptors (PPARs) activate genes related to fat oxidation and proliferation of peroxisomes in the liver through 
induction of PEX proteins (60). Components of milk, specifically milk fats, stimulate the activation of PPARs (61, 62). Therefore, there is the potential that peroxisomal deficiency or dysfunction resulted in the build of cerotic (C26:0), nonadecanoic (C19:0), Lyso-PG(20:5), in about one-third of the IF gilts, and this was due to IF gilts consuming less milk than HF gilts.

To explore this as a potential mechanism, we queried data from Rahman et al., where they evaluated the effects of exposure to colostrum versus milk replacer on the uterine transcriptome (63). Briefly, for their study, gilts were either allowed to nurse from sows ad libitum or were bottle fed a commercial milk replacer for 48 hours after birth. Animals for euthanized at $48 \mathrm{~h}$ postnatal, uterine tissues removed and RNA was isolated for transcriptome analysis using RNA-seq (63). Transcriptome data were searched for peroxisomal related genes, and we found that peroxisomal biogenesis factor 5 like (PEX5L), acyl-coenzyme A 3 (ACOX3), peroxisome proliferator activated receptor alpha (PPARa), and arachidonate 15-lipoxygenase (ALOX15) were numerically, but not significantly, higher in gilts that nursed sow's milk versus replacer-fed gilts. PEX5L is a paralog to PEX5 which is responsible for the assembly and function of peroxisomes (64). Mice with knockout for PEX5 have an accumulation of VLCFAs due to the inability to transport VLCFAs into the peroxisome and a disruption of the PEX5 gene is common in Zellweger Syndrome patients (65). Another gene associated with Zellweger syndrome is ACOX3. ACOX3 functions in the regulation of peroxisomal lipid metabolism and is considered the rate-limiting step in the degradation of methyl branched fatty acids (66). PPARa is transcription factor that regulates genes involved in lipid degradation and inflammation, where PPARa is primarily responsible for the degradation of inflammatory fatty acid residues (67). Lastly, ALOX15 acts on a number of PUFAs to synthesize lipid mediators such as eicosanoids and also regulates inflammation (64). These data show that piglets exposed to colostrum had greater levels of expression of peroxisome related genes.

Metabolome-lipidome profiles are reflective of what has happened in a system. Vaginal tissue is composed of stratified epithelium. The stratification of the cells and movement from basal to apical layers reflects a history of exposures. Our studies of the vagina showed that the layers of stratification increased in parallel with alterations in abundance of proteins as the tissue developed over the first two weeks postnatal (68), and thus samples taken at two and three weeks postnatal likely reflect biochemical products related to nutritional environment in the perinatal period. Beta-oxidation of VLCFA and break-down of odd chain fatty acids and eicosanoid derivatives occur in peroxisomes (60). Infertile animals at weaning had a greater abundance of VLCFA, eicosanoids and odd chain fatty acids and low DHA and ARA in cellular material captured with vaginal swabs. We hypothesize that the low AA and DHA were due to lower levels of colostrum intake, and the build-up of VLCFA, odd-chain fatty acids and PUFA reflected a factor in infertile animal's perinatal environment that affected peroxisome function or biogenesis (69).

Approximately one-third of the IF animals were distinguished in principle component analysis of FFA and lipids in Method 1. These animals were also driving the associations between arachidonic acid and cerotic acid, ximenic acid, and the Lyso PG lipids. These data indicate that while all infertile animals did not show distinct lipidomes, likely reflecting multiple factors that affect fertility, approximately one-third showed lipidome profiles at weaning that distinguished them from highly fertile animals. Identification of one-third of the animals in a herd that are infertile at 21 days postnatal has the potential to have a large financial impact for the producer, as they would greatly reduce the overall number of resources used for a group that provides minimal economic return.

\subsection{Conclusions}


Low ARA combined with higher abundance of very long chain fatty acids and phospholipids with prostaglandins in vaginal swabs taken at weaning was predictive of infertility in approximately one-third of IF animals in this study. ROC curve analysis supports further exploration of cerotic acid (C26:0), ximenic acid (C26:1) and nonadecanoic acid (C19:0) as potential biomarkers of reproductive efficiency, as well as studies aimed at understanding the mechanistic relationship of a greater proportion of long chain fatty acids and higher proportion of PUFA in vaginal swabs of three-week old piglets that in the long-term are infertile. Application of these findings has the potential to improve reproductive efficiency of swine farms, and in this way reduce economic losses for the producer. The potential association of findings between piglet colostrum-milk intake, peroxisome biogenesis and reproductive failure at puberty warrants future research.

\subsection{Abbreviations}

AC: Acylcarnitine

ACOX3: Acyl-Coenzyme A 3

ALD: adrenoleukodystrophy

ALA: Alpha linoleic acid

ALOX15: Arachidonate 15-Lipoxygenase

ARA: Arachidonic Acid

AUC: Area-under-the-curve

Al: Artificial Insemination

CE: Cholesterol ester

DHA: Docohexaenoic acid

FFA: Free fatty acids

LA: Linoleic acid

LPG: Lysophosphatidylglycerol

MRM: Multiple reaction monitoring

PC: Phosphatidylcholines

PCo: Phosphatidylcholine O-

PCOS: Polycystic ovary syndrome

PE: Phosphatidylethanolamines

PEX: Peroxin 
PEX5L: Peroxisomal biogenesis factor 5 like

PG: Phosphatidylglycerols

PGF2a: Prostaglandin-F2a

Pl: Phosphatidylinositols

PPAR: Peroxisome proliferator activator receptors

PN: Postnatal

PS: Phosphatidylserines

PSo: Phosphatidylserines 0-

PSY: Pigs weaned per sow per ear

PUFA: Polyunsaturated fatty acids

ROC: Receiver operating characteristic

SM: Sphingomyelin

TAG: Triacylglycerols

ULCFA: Ultra long-chain fatty acids

VLCFA: Very long-chain fatty acids

\subsection{Declarations}

\subsubsection{Ethics approval and consent to participate}

All animal procedures were approved by the Institutional Animal Care and Use Committee at Purdue University.

\subsubsection{Consent for publication}

No human data was collected for this study.

\subsubsection{Availability of data and material}

Detailed lipidomic data is provided in supplemental materials with this publication.

\subsubsection{Competing interests}

The authors declare that they have no competing interests.

\subsubsection{Funding}

Funding for this work was provided by the National Pork Board grant no. 17-153. 


\subsubsection{Author's contributions}

$\mathrm{KM}$ collected and analyzed data as well as writing the manuscript. CF performed lipidomic analysis and edited the manuscript. JS oversaw live-animal data collection. KS performed study design, data interpretation and edited the manuscript. TC oversaw the entire project, interpretation and writing.

\subsubsection{Acknowledgements}

All lipidome MRM-profiling was performed at the Purdue Metabolomics Core Facility in the Bindley Bioscience Center. We are also grateful to Tiago J. P. Sobreira for the data processing support, and to Ag Production Enterprises, Inc. for allowing this study to be held at their facility.

\subsection{References}

1. Stalder KJ, Lacy RC, Cross TL, Conatser GE. Financial impact of average parity of culled females in a breedto-wean swine operation using replacement gilt net present value analysis. Journal of Swine Health and Production. 2003;11(2):69-74.

2. Stalder KJ. Calculating Payback Parity for Replacement Gilts: National Hog Farmer; 2009 [Available from: http://www.nationalhogfarmer.com/genetics-reproduction/0109-calculating-payback-parity.

3. Stalder KJ, Duttlinger V, Marsh W. A better method to measure sow herd efficiency 2019 [Available from: https://www.pigprogress.net/Sows/Articles/2019/1/Is-there-one-method-fits-all-to-measure-sow-herdefficiency-378970E/.

4. PigCHAMP. USA 2018 Year Summary 2019 [Available from: https://www.pigchamp.com/news/benchmarkmagazine/articles/usa-2018-year-summary-2019.

5. Patterson J, Beltranena E, Foxcroft G. The effect of gilt age at first estrus and breeding on third estrus on sow body weight changes and long-term reproductive performance. Journal of animal science. 2010;88(7):250013.

6. Graves KL, Mordhorst BR, Wright EC, Hale BJ, Stalder KJ, Keating AF, et al. Identification of measures predictive of age of puberty onset in gilts. Translational Animal Science. 2020;4(1):285-92.

7. Romoser MR, Hale BJ, Seibert JT, Gall T, Rademacher CJ, Stalder KJ, et al. Methods for reproductive tract scoring as a tool for improving sow productivity. Translational Animal Science. 2020;4(1):275-84.

8. Mills KM, Schinckel AP, Stevens JG, Casey TM, Stewart KR. Evaluation of on-farm indicators of gilt reproductive performance potential at 21 days of age. Translational Animal Science. 2020.

9. Bagnell CA, Steinetz BG, Bartol FF. Milk-borne relaxin and the lactocrine hypothesis for maternal programming of neonatal tissues. Annals of the New York Academy of Sciences. 2009;1160(1):152-7.

10. Bartol F, Wiley A, Spencer T, Vallet J, Christenson R. Early uterine development in pigs. JOURNAL OF REPRODUCTION AND FERTILITY-SUPPLEMENT-. 1993:99-.

11. Bartol FF, Wiley AA, Bagnell CA. Relaxin and maternal lactocrine programming of neonatal uterine development. Annals of the New York Academy of Sciences. 2009;1160(1):158-63.

12. Bagnell CA, Bartol FF. Relaxin and the 'Milky Way': The lactocrine hypothesis and maternal programming of development. Molecular and cellular endocrinology. 2019;487:18-23. 
13. Bartol F, Wiley A, Bagnell C. Uterine development and endometrial programming. Society of Reproduction and Fertility supplement. 2006;62:113-30.

14. Vallet J, Miles J, Rempel L, Nonneman D, Lents C. Relationships between day one piglet serum immunoglobulin immunocrit and subsequent growth, puberty attainment, litter size, and lactation performance. Journal of animal science. 2015;93(6):2722-9.

15. Harlow K, Ferreira CR, Sobreira TJ, Casey T, Stewart K. Lipidome profiles of postnatal day 2 vaginal swabs reflect fat composition of gilt's postnatal diet. PloS one. 2019;14(9).

16. Casey T, Harlow K, Ferreira CR, Sobreira TJ, Schinckel A, Stewart K. The potential of identifying replacement gilts by screening for lipid biomarkers in reproductive tract swabs taken at weaning. Journal of Applied Animal Research. 2018;46(1):667-76.

17. Koketsu Y, Tani S, lida R. Factors for improving reproductive performance of sows and herd productivity in commercial breeding herds. Porcine Health Management. 2017;3(1):1.

18. Vinsky M, Novak S, Dixon W, Dyck M, Foxcroft G. Nutritional restriction in lactating primiparous sows selectively affects female embryo survival and overall litter development. Reproduction, Fertility and Development. 2006;18(3):347-55.

19. Soede N, Langendijk P, Kemp B. Reproductive cycles in pigs. Animal reproduction science. 2011;124(3-4):2518.

20. Bligh EG, Dyer WJ. A rapid method of total lipid extraction and purification. Canadian journal of biochemistry and physiology. 1959;37(8):911-7.

21. de Lima CB, Ferreira CR, Milazzotto MP, Sobreira TJP, Vireque AA, Cooks RG. Comprehensive lipid profiling of early stage oocytes and embryos by MRM profiling. Journal of mass spectrometry: JMS. 2018;53(12):124752.

22. Dipali SS, Ferreira CR, Zhou LT, Pritchard MT, Duncan FE. Histologic analysis and lipid profiling reveal reproductive age-associated changes in peri-ovarian adipose tissue. Reproductive Biology and Endocrinology. 2019;17(1):1-13.

23. O'Neil EV, Burns GW, Ferreira CR, Spencer TE. Characterization and regulation of extracellular vesicles in the lumen of the ovine uterus. Biology of reproduction. 2020;102(5):1020-32.

24. Suarez-Trujillo A, Huff K, Ramires Ferreira C, Paschoal Sobreira TJ, Buhman KK, Casey T. High-fat-diet induced obesity increases the proportion of linoleic acyl residues in dam serum and milk and in suckling neonate circulation. Biology of Reproduction. 2020;103(4):736-49.

25. Harlow K, Ferreira CR, Sobreira TJ, Casey T, Stewart K. Lipidome profiles of postnatal day 2 vaginal swabs reflect fat composition of gilt's postnatal diet. PloS one. 2019;14(9):e0215186.

26. Pang Z, Chong J, Li S, Xia J. MetaboAnalystR 3.0: Toward an Optimized Workflow for Global Metabolomics. Metabolites. 2020;10(5):186.

27. Xia J, Broadhurst DI, Wilson M, Wishart DS. Translational biomarker discovery in clinical metabolomics: an introductory tutorial. Metabolomics. 2013;9(2):280-99.

28. Nehra D, Le HD, Fallon EM, Carlson SJ, Woods D, White YA, et al. Prolonging the female reproductive lifespan and improving egg quality with dietary omega-3 fatty acids. Aging cell. 2012;11(6):1046-54.

29. Elis S, Freret S, Desmarchais A, Maillard V, Cognié J, Briant E, et al. Effect of a long chain n-3 PUFA-enriched diet on production and reproduction variables in Holstein dairy cows. Animal reproduction science. 
2016;164:121-32.

30. Sinedino LD, Honda PM, Souza LR, Lock AL, Boland MP, Staples CR, et al. Effects of supplementation with docosahexaenoic acid on reproduction of dairy cows. Reproduction. 2017;153(5):707-23.

31. Maillard V, Desmarchais A, Durcin M, Uzbekova S, Elis S. Docosahexaenoic acid (DHA) effects on proliferation and steroidogenesis of bovine granulosa cells. Reproductive Biology and Endocrinology. 2018;16(1):40.

32. Smit M, Patterson JL, Spencer JD, Dixon WT, Dyck MK, Foxcroft GR, et al. Responses to n-3 fatty acid (LCPUFA) supplementation of gestating gilts, and lactating and weaned sows. 2012.

33. Whelan J, Fritsche K. Linoleic Acid. Advances in Nutrition. 2013;4(3):311-2.

34. Arbuckle LD, Innis SM. Docosahexaenoic Acid Is Transferred through Maternal Diet to Milk and to Tissues of Natural Milk-Fed Piglets. The Journal of Nutrition. 1993;123(10):1668-75.

35. Lin LE, Chen CT, Hildebrand KD, Liu Z, Hopperton KE, Bazinet RP. Chronic dietary n-6 PUFA deprivation leads to conservation of arachidonic acid and more rapid loss of DHA in rat brain phospholipids. J Lipid Res. 2015;56(2):390-402.

36. Roszkos R, Tóth T, Mézes M. Practical Use of n-3 Fatty Acids to Improve Reproduction Parameters in the Context of Modern Sow Nutrition. Animals. 2020;10(7):1141.

37. Davis TA, Nguyen HV, Garcia-Bravo R, Fiorotto ML, Jackson EM, Lewis DS, et al. Amino Acid Composition of Human Milk Is Not Unique. The Journal of Nutrition. 1994;124(7):1126-32.

38. Bilby T, Guzeloglu A, MacLaren L, Staples C, Thatcher W. Pregnancy, bovine somatotropin, and dietary n-3 fatty acids in lactating dairy cows: II. Endometrial gene expression related to maintenance of pregnancy. Journal of dairy science. 2006;89(9):3375-85.

39. Tang B, Shang X, Qi H, Li J, Ma B, An G, et al. Metabonomic analysis of fatty acids in seminal plasma between healthy and asthenozoospermic men based on gas chromatography mass spectrometry. Andrologia. 2017;49(9):e12744.

40. Jenkins B, West JA, Koulman A. A review of odd-chain fatty acid metabolism and the role of pentadecanoic acid (C15: 0) and heptadecanoic acid (C17: 0) in health and disease. Molecules. 2015;20(2):2425-44.

41. Leon HA, Feller DD, Neville ED, Daligcon B. Stress Induced Stimulation of Liver Protein Synthesis Independent of Adrenal or Pituitary Action. Life Sci. 1965;62:737-41.

42. Kim S, Chen J, Cheng T, Gindulyte A, He J, He S, et al. PubChem 2019 update: improved access to chemical data. Nucleic acids research. 2019;47(D1):D1102-D9.

43. Sassa T, Kihara A. Metabolism of very long-chain Fatty acids: genes and pathophysiology. Biomol Ther (Seoul). 2014;22(2):83-92.

44. Proksch E, Brandner JM, Jensen JM. The skin: an indispensable barrier. Experimental dermatology. 2008;17(12):1063-72.

45. Whigham L, Butz D, Dashti H, Tonelli M, Johnson L, Cook M, et al. Metabolic evidence of diminished lipid oxidation in women with polycystic ovary syndrome. Current Metabolomics. 2013;1(4):269-78.

46. Steinberg SJ, Dodt G, Raymond GV, Braverman NE, Moser AB, Moser HW. Peroxisome biogenesis disorders. Biochimica et Biophysica Acta (BBA)-Molecular Cell Research. 2006;1763(12):1733-48.

47. Moser HW, Mahmood A, Raymond GV. X-linked adrenoleukodystrophy. Nature Clinical Practice Neurology. 2007;3(3):140-51. 
48. Lu J-F, Lawler AM, Watkins PA, Powers JM, Moser AB, Moser HW, et al. A mouse model for X-linked adrenoleukodystrophy. Proceedings of the National Academy of Sciences. 1997;94(17):9366-71.

49. Powers JM, Schaumburg HH. The testis in adreno-leukodystrophy. Am J Pathol. 1981;102(1):90-8.

50. Rodriguez-Estrada M, Cilla Tatai A, Cardenia V, Garcia-Llatas G. Fats and Sterols. 2014.

51. Belaz KR, Tata A, França MR, Santos da Silva MI, Vendramini PH, Fernandes AM, et al. Phospholipid Profile and Distribution in the Receptive Oviduct and Uterus During Early Diestrus in Cattle. Biol Reprod. 2016;95(6):127.

52. Diczfalusy U, Kase B, Alexson S, Björkhem I. Metabolism of prostaglandin F2 alpha in Zellweger syndrome. Peroxisomal beta-oxidation is a major importance for in vivo degradation of prostaglandins in humans. The Journal of clinical investigation. 1991;88(3):978-84.

53. Luzzo KM, Wang Q, Purcell SH, Chi M, Jimenez PT, Grindler N, et al. High fat diet induced developmental defects in the mouse: oocyte meiotic aneuploidy and fetal growth retardation/brain defects. PloS one. 2012;7(11):e49217.

54. Minge CE, Bennett BD, Norman RJ, Robker RL. Peroxisome proliferator-activated receptor-y agonist rosiglitazone reverses the adverse effects of diet-induced obesity on oocyte quality. Endocrinology. 2008;149(5):2646-56.

55. Jungheim ES, Schoeller EL, Marquard KL, Louden ED, Schaffer JE, Moley KH. Diet-induced obesity model: abnormal oocytes and persistent growth abnormalities in the offspring. Endocrinology. 2010;151(8):4039-46.

56. Wu LL-Y, Dunning KR, Yang X, Russell DL, Lane M, Norman RJ, et al. High-fat diet causes lipotoxicity responses in cumulus-oocyte complexes and decreased fertilization rates. Endocrinology. 2010;151(11):5438-45.

57. Dennis EA, Norris PC. Eicosanoid storm in infection and inflammation. Nature Reviews Immunology. 2015;15(8):511-23.

58. Poulos A, Beckman K, Johnson D, Paton B, Robinson B, Sharp P, et al. Very long-chain fatty acids in peroxisomal disease. Neurobiology of Essential Fatty Acids: Springer; 1992. p. 331-40.

59. Distel B, Erdmann R, Gould SJ, Blobel G, Crane DI, Cregg JM, et al. A unified nomenclature for peroxisome biogenesis factors. The Journal of Cell Biology. 1996;135(1):1-3.

60. Lodhi IJ, Semenkovich CF. Peroxisomes: a nexus for lipid metabolism and cellular signaling. Cell metabolism. 2014;19(3):380-92.

61. Parodi PW. Cooperative action of bioactive components in milk fat with PPARs may explain its antidiabetogenic properties. Medical Hypotheses. 2016;89:1-7.

62. Shi B, Zhao X, Wang C, Wang N, Tian M, Shan A. I-carnitine and fat type in the maternal diet during gestation and lactation modify the fatty acid composition and expression of lipid metabolism-related genes in piglets. Journal of animal physiology and animal nutrition. 2019;103(4):1207-17.

63. Rahman KM, Camp ME, Prasad N, McNeel AK, Levy SE, Bartol FF, et al. Age and Nursing Affect the Neonatal Porcine Uterine Transcriptome1. Biology of Reproduction. 2016;94(2).

64. Stelzer G, Rosen N, Plaschkes I, Zimmerman S, Twik M, Fishilevich S, et al. The GeneCards Suite: From Gene Data Mining to Disease Genome Sequence Analyses. Current protocols in bioinformatics. 2016;54(1):1.30.11..3. 
65. Baumgart E, Vanhorebeek I, Grabenbauer M, Borgers M, Declercq PE, Fahimi HD, et al. Mitochondrial alterations caused by defective peroxisomal biogenesis in a mouse model for Zellweger syndrome (PEX5 knockout mouse). The American journal of pathology. 2001;159(4):1477-94.

66. Hunt MC, Siponen MI, Alexson SE. The emerging role of acyl-CoA thioesterases and acyltransferases in regulating peroxisomal lipid metabolism. Biochimica et Biophysica Acta (BBA)-Molecular Basis of Disease. 2012;1822(9):1397-410.

67. Chinetti G, Fruchart J-C, Staels B. Peroxisome proliferator-activated receptors (PPARs): nuclear receptors at the crossroads between lipid metabolism and inflammation. Inflammation research. 2000;49(10):497-505.

68. Harlow K, Suarez-Trujillo A, Hedrick V, Sobreira T, Aryal UK, Stewart K, et al. Temporal analysis of vaginal proteome reveals developmental changes in lower reproductive tract of gilts across the first two weeks postnatal. Scientific reports. 2019;9(1):13241.

69. Farr RL, Lismont C, Terlecky SR, Fransen M. Peroxisome biogenesis in mammalian cells: The impact of genes and environment. Biochimica et Biophysica Acta (BBA) - Molecular Cell Research. 2016;1863(5):104960.

\section{Figures}

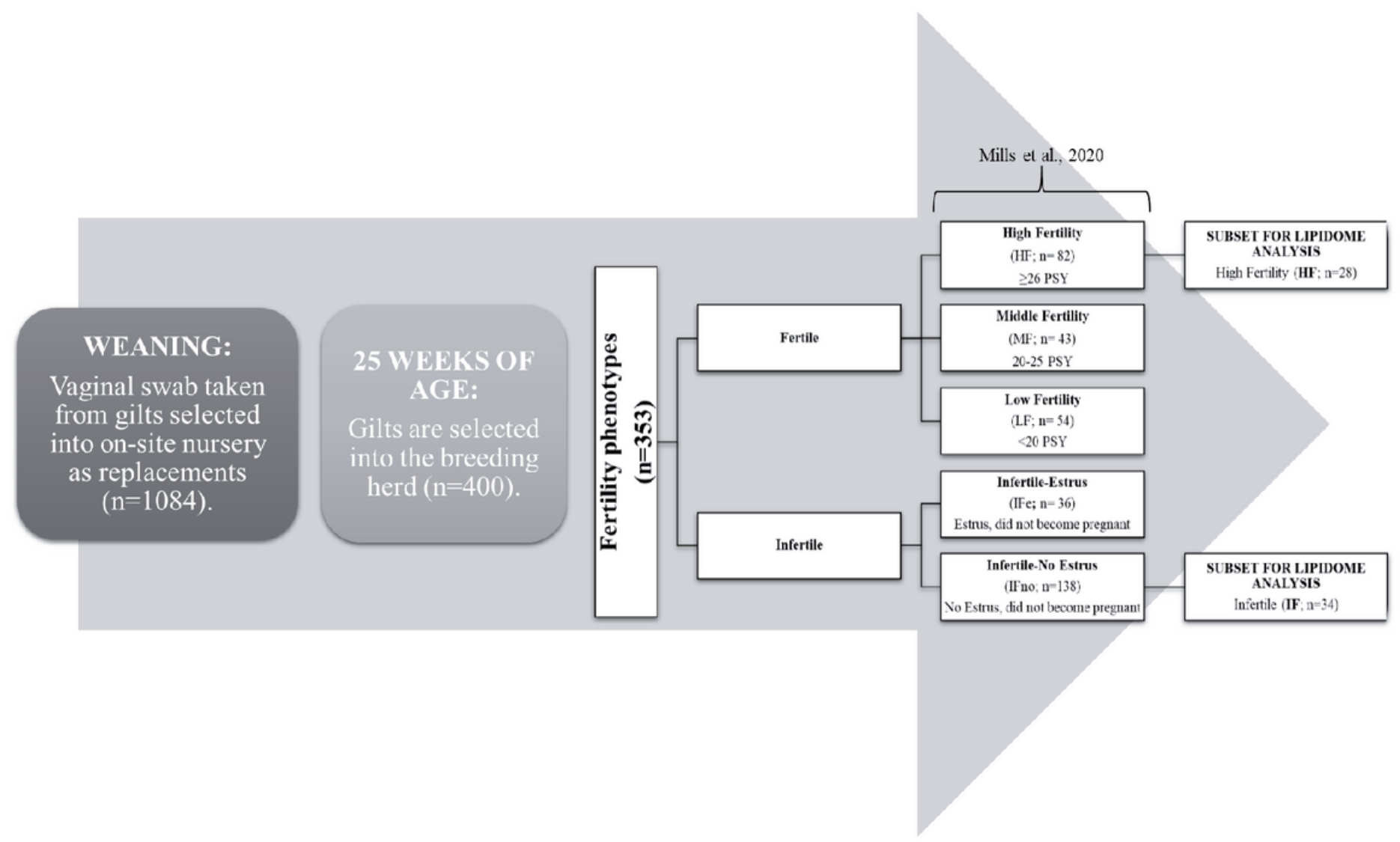

\section{Figure 1}

Longitudinal study design and fertility classification. This figure depicts the timeline of the study highlighting the three stages of replacement sow selection and characterization of fertility phenotypes. Fertility phenotypes were 
characterized based on pigs per sow per year (PSY) and whether gilts exhibited signs of estrus. A subset of animals from the most extreme fertility phenotypes previously described in Mills et al., 2020 were used for lipidomic analysis. Gilts classified as infertile for lipidome analysis never exhibited estrus and were never bred. Fertile gilts selected for lipidome analysis were from the high fertility group where gilts had at least 26 PSY.
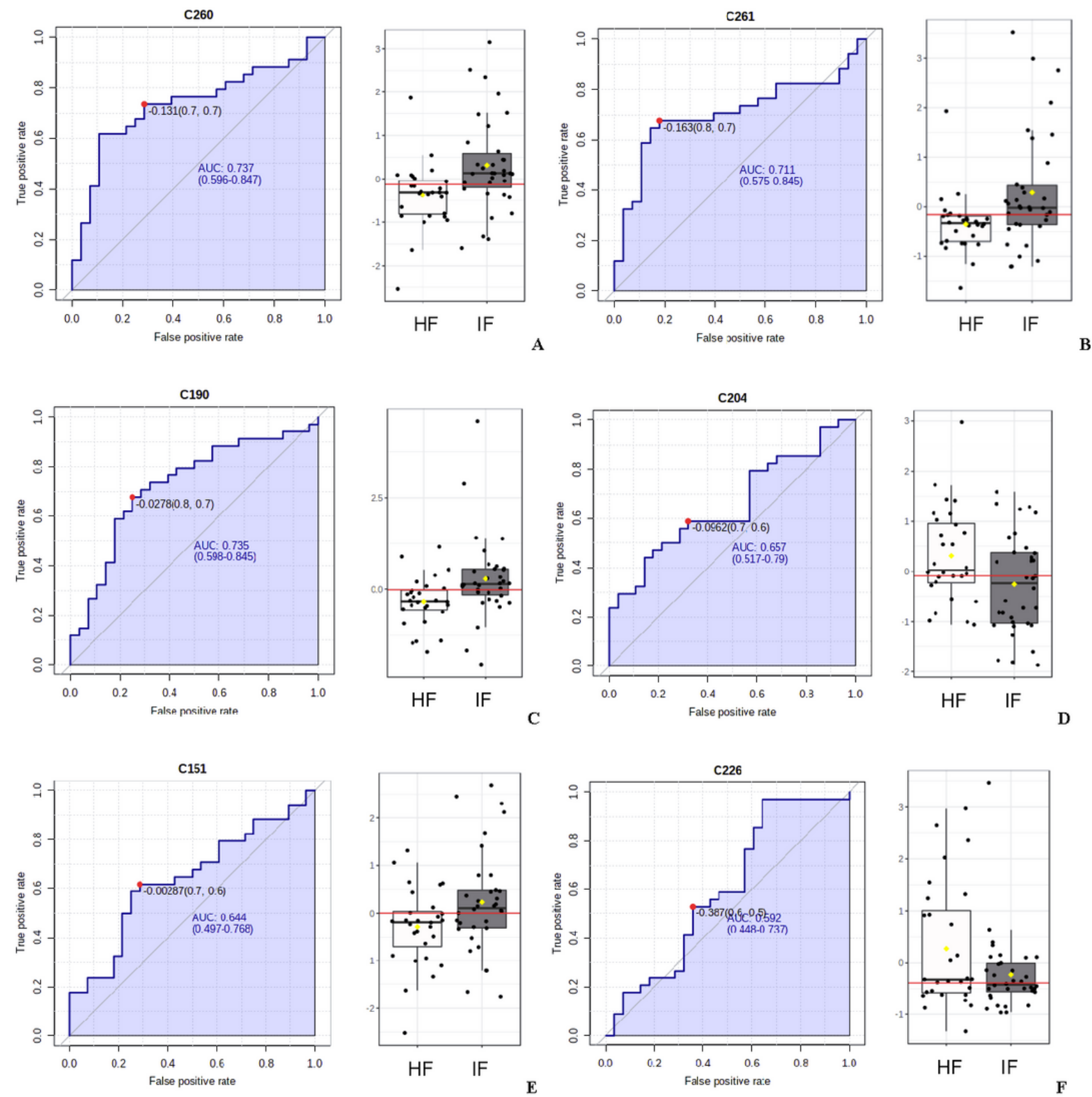

\section{Figure 2}

ROC curve analysis of differentially abundant FFAs. (A) Area-under-the-curve (AUC) value and distribution of high fertility (HF) versus infertile animals (IF) for C(26:0);Cerotic Acid. (B) C(26:1);Ximenic Acid (C) C(19:0);Nonadecanoic Acid (D) C(20:4);Arachidonic Acid (E) C(15:1);Pentadecenoic Acid and (F) $\mathrm{C}(22: 6) ;$ Docosahexaenoic Acid. 

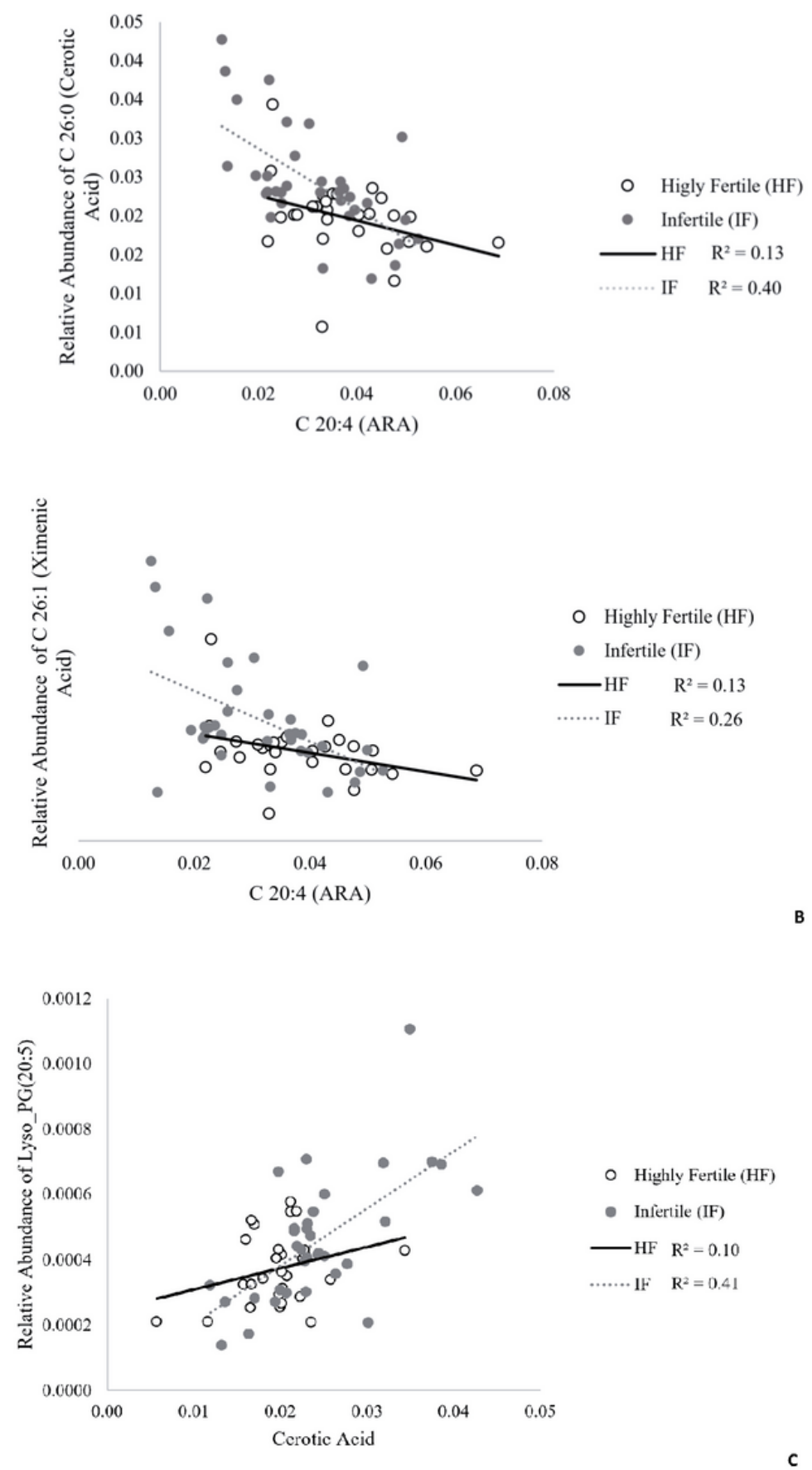

c

\section{Figure 3}

Correlation analysis of distinguishing free fatty acids and glycerophosphoglycerols within IF and HF animals. (A) Correlation analysis of the relative abundance of $\mathrm{C}(26: 0)$ Cerotic Acid and Arachadonic acid (ARA). (B) Correlation analysis of the relative abundance of $\mathrm{C}(26: 1)$ Ximenic Acid and ARA. (C) Correlation analysis of the relative abundance of Lyso_PG (20:5) and Cerotic acid.

\section{Supplementary Files}

This is a list of supplementary files associated with this preprint. Click to download. 
- JASBAdditionalFiles.xlsx 